Document downloaded from:

http://hdl.handle.net/10251/64901

This paper must be cited as:

Aguado Sarrió, E.; Prats-Montalbán, JM.; Sanz Requena, R.; Marti Bonmati, L.; Alberich Bayarri, A.; Ferrer Riquelme, AJ. (2015). Prostate Diffusion Weighted-Magnetic Resonance Image analysis using Multivariate Curve Resolution methods. Chemometrics and Intelligent Laboratory Systems. 140:43-48. doi:10.1016/j.chemolab.2014.11.002.

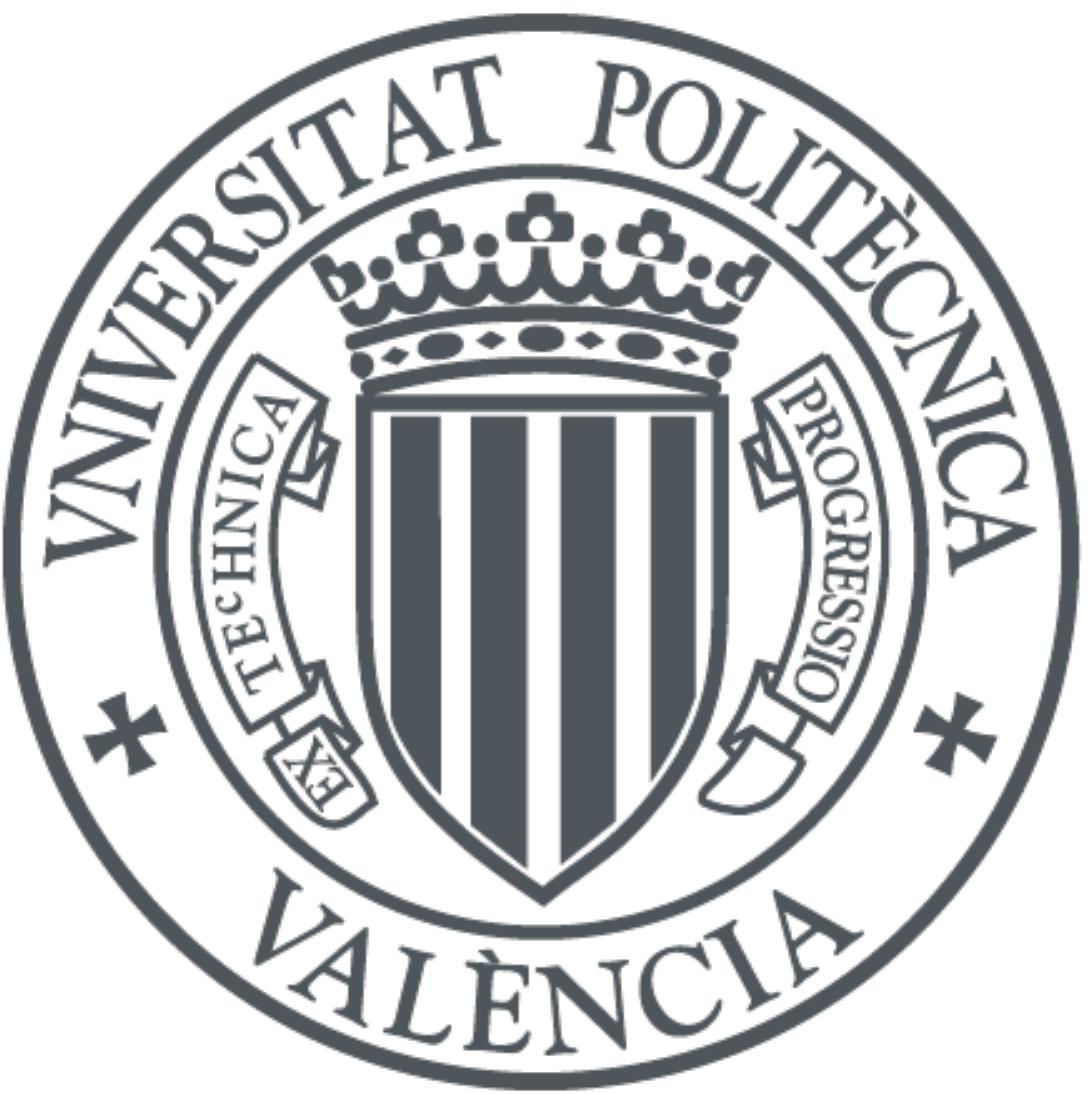

The final publication is available at

https://dx.doi.org/10.1016/j.chemolab.2014.11.002

Copyright Elsevier

Additional Information 


\title{
Prostate Diffusion Weighted-Magnetic Resonance Image analysis using Multivariate Curve Resolution Methods
}

\author{
Aguado-Sarrió, E. ; Prats-Montalbán, J.M. ${ }^{\mathrm{a}^{*}}$, Sanz-Requena, R. ${ }^{\text {b,c }}$, Martí-Bonmatí, L. ${ }^{\mathrm{c}, \mathrm{d}}$, \\ Alberich-Bayarri, A. ${ }^{\mathrm{c}}$ and Ferrer, A. ${ }^{\mathrm{a}}$
}

a) Multivariate Statistical Engineering Group, Universitat Politècnica de València, Valencia, Spain

b) Biomedical Engineering, Hospital Quirón Valencia, Valencia, Spain c) Biomedical Imaging Research Group (GIBI230), Hospital Universitario y Politécnico La Fe, Valencia, Spain

d) Radiology Department, Hospital Quirón Valencia, Valencia, Spain

* Corresponding author: José M. Prats-Montalbán. Departamento de Estadística e IO Aplicadas y Calidad. Universidad Politécnica de Valencia. Cno. De Vera s/n, Edificio 7A, 46022, Valencia, Spain. Tlf: +34.96.387.70.07 ext. 74949, Fax: +34.96.387.74.99. E-mail: jopramon@eio.upv.es

\begin{abstract}
Multivariate Curve Resolution (MCR) has been applied on prostate Diffusion Weighted - Magnetic Resonance Images (DW-MRI). Different physiological-based modeling approaches of the diffusion process have been submitted to validation by sequentially incorporating prior knowledge on the MCR constraints. Results validate the biexponential diffusion modeling approach and show the capability of the MCR models to find, characterize and locate the behaviors related to the presence of an early prostate tumor.
\end{abstract}




\section{INTRODUCTION}

Two of the main indicators of a tumor process are the neovascularization and the increase in cellular density. When a group of growing cells presents abnormally high demands of oxygen and nutrients, the tissue responds by creating new vessels (angiogenesis) or developing existing ones (neovascularization). On the other hand, the biological process associated with higher cellular densities that leads to cell agglomeration in the tissue is called cellularization. The combination of both processes is what usually determines the presence of an early tumor as first steps in oncogenesis. One way to approach this combination is by studying the tissue local diffusion process [1], which is a physical process that occurs due to the thermal agitation of the water molecules inside the human body. These translational displacements depend, among other factors, on the tissue structure according to the cellular organization. When the tissue is highly cellularized, the molecules have more restrictions to movement due to a decreased interstitial space and higher cell membrane interfases. However, when the tissue is highly vascularized, molecules are in a non-restricted high velocity environment within the vessels, and the spatial movements are random with less restrictions in all spatial directions.

The diffusion process can be evaluated with a Diffusion-Weighted Magnetic Resonance Imaging (DW-MRI). This non-invasive technique provides high resolution images that are sensitive to water molecules movement inside the tissues. Depending on the configuration of the MR equipment and based on the duration and the amplitude of the applied magnetic field gradient, image acquisition is associated to a parameter known as $b$-value [2]. The signal of the image decreases with the increase in the b-value acquired. This attenuation depends on the characteristics of the tissue, being stronger if the tissue is vascularized and much more moderate if it is highly cellular. The range of different signal attenuations between these two types of tissue at the same b-value is the basics to study the different behaviors in the diffusion process.

The DW-MRI acquisition sequence is performed along the volume of the studied organ. Usually, images are acquired at spatial planes corresponding to different slices of the human body (the number of slices depends on the studied organ). For each slice, images are taken with different b-values, obtaining a 3D data structure. This way, a signal 
spectrum $\mathbf{s}$ is extracted from each pixel of the image, associated to the different $b$ values. The number of $b$-values varies among clinical studies, reaching up to 10 values for the clinical setting [3]. In our study, $6 \mathrm{~b}$-values were used for prostate imaging based on previous experience.

In order to model the signal decay of the diffusion process, spectra can be fitted with different expressions. The most widely used model in clinical routine is the monoexponential diffusion model [2] with the apparent diffusion coefficient (ADC) as its parameter:

$$
\frac{s}{s_{0}}=e^{-b(A D C)}
$$

Where $\mathrm{s}_{0}$ is the initial value of the signal when the $b$-value equals zero. The ADC values express the average distance that the water molecules cover within a voxel at a certain time. It is related with the cell density, the permeability of the membranes and the tortuosity of the intercellular interstitial space. It is called "apparent" because it reflects several different mechanisms, as it is a combination of the two phenomena expressed before: (i) the movement associated to the water molecules (Brownian movement), known as slow diffusion (cellular tissue), and (ii) the intravascular movement in the microcapillaries vessels, known as fast diffusion or perfusion (vascularized tissue). Fast diffusion may produce an overestimation of the real diffusion values if not properly considered.

The monoexponential model does not take into account the different mechanisms of the diffusion process. Currently, one way of dealing with this complexity is by using a biexponential model. This is a more complex model that considers two behaviors, slow and fast diffusion, weighted with a new parameter called vascular fraction $(f)$, which relates to the proportion of vascular tissue in a voxel. This model is also known as intravoxel incoherent motions (IVIM) [4] due to the two types of movements considered, related to cellularity (slow diffusion) and vascularization (fast diffusion). The expression of the IVIM model is shown below.

$$
\frac{s}{s_{0}}=(1-f) e^{-b D}+f e^{-b\left(D+D^{*}\right)}
$$


The spectra are normalized with $s_{0}$ as in the monoexponential approach. Three different parameters must be estimated: the diffusion coefficient $(D)$, the pseudo-perfusion coefficient $\left(D^{*}\right)$ and the vascular fraction $(f)$. This way, the normalized signal $\mathrm{s} / \mathrm{s}_{0}$ is modeled as a weighted average of the slow diffusion (water movement inside the cellular tissue, characterized by $D$ ) and the fast diffusion (water movement inside the vascular tissue, characterized by $D+D^{*}$ ). The slow diffusion behavior is weighted by $(1-f)$ and the fast diffusion term by $f$, since the major contribution of this phase (in the order of 10 times higher) is from $\mathrm{D}^{*}$ if compared to $\mathrm{D}$, however this last parameter is not so low to be obviated. Despite the IVIM model is theoretically more appropriate according to physiological criteria, the monoexponential model is, nowadays, the most widely used in medical practice to model the diffusion process.

Besides, the IVIM model is not a classic biexponential model because the two exponential decays are not independent as they are complementary weighted by the vascular fraction, $f$. Furthermore, the normalization of the spectra causes a distortion, modifying the shape of the original curve (Fig. 1a) and decreasing the signal-to-noise ratio (as can be seen in Fig. 1b). Thus, this standardization reduces the variability range masking the different behaviors present in the spectra. All these concepts shown above and the difficulty in the interpretation of the results provided by these biomarkers (such as $D, D^{*}$ and $f$ parameters) have limited their applicability in clinical practice.

\section{[INSERT FIG. 1 ABOUT HERE]}

Furthermore, these biomarkers are obtained from a generally pixel-by-pixel modeling, which do not take advantage of the relation between pixels with the same behavior, increasing the uncertainty in their estimation; and degrading the corresponding imaging biomarkers (images built from each $D, D^{*}$ and $f$ parameters at each pixel location) used for clinical purposes.

One possible alternative to analyze these diffusion behaviors is by applying multivariate statistical models, so that it is possible to take advantage of the relation between pixels. When dealing with images, the application of these types of models is known as Multivariate Image Analysis (MIA) [5,6]. The main characteristic of MIA is the 
capability to study the whole set of pixels at the same time by extracting the sources of variation caused by the latent structures present in the images. In this way, MIA can help in providing new non-parametric models that can explain the principal diffusion behaviors extracted from DW-MRI. It may also be useful to check the appropriateness of the different modeling alternatives (e.g. monoexponential or biexponential) proposed in the literature.

The main and most widespread MIA tool is PCA (Principal Component Analysis) [7]. However, two problems arise when PCA is applied on DW-MRI data: (i) no prior information can be included in the model, and (ii) the orthogonality of the principal components is a limitation to model the different diffusion behaviors that are not necessarily orthogonal. In order to overcome these drawbacks, it is possible to use more flexible models, as is the case of Multivariate Curve Resolution (MCR), which has been already applied very recently to dynamic contrast-enhanced MRI data [8].

The goals of this work are: (i) to explore the capability of MCR methods to model the different behaviors associated to the diffusion process in DW-MRI helping specialists to detect and characterize early tumors in the prostate, (ii) to check the adequacy of the different theoretical models commonly applied in clinical practice, by sequentially incorporating constraints in the MCR algorithm using prior knowledge about the diffusion process, (iii) to provide new imaging biomarkers that may complement those commonly used for clinical diagnosis.

\section{MATERIALS AND METHODS}

The database consists of DW-MRI acquired from 10 patients with proven prostate carcinoma. The images for each patient were taken along 12 slices covering the whole prostate. For each slice, images with a resolution of 192x192 pixels were acquired with 6 different b-values $\left(0,50,200,400,1000\right.$ and $\left.2000 \mathrm{~s} / \mathrm{mm}^{2}\right)$ and arranged in a 3D matrix $(192 \times 192 \times 6)($ see Fig. $2 a)$. The output for each patient was a series of twelve 3D images. All images were anonymized and transferred to a dedicated workstation for post-processing. 
In order to analyze the images by latent-based bilinear multivariate statistical models, the 3D matrix for each slice was unfolded keeping the b-values mode yielding a 2D matrix $(36864 \times 6)$ that contains all the pixels for each slice in rows and the different $b$ values in columns (see Fig. 2 right). All the slices from the same patient were studied with the same model by stacking the unfolded 2D matrices of each slice one below the other obtaining a data matrix $\mathbf{S}(442368 \times 6)$. This way the fitted behaviors were forced to keep the same internal correlation structure along the whole prostate volume for a particular patient.

\section{[INSERT FIG. 2 ABOUT HERE]}

In order to focus the study in the prostate gland, local models were built for each of the 10 analyzed cases by removing the pixels that do not pertain to the prostate zone with manual masks provided by the doctors. This way, the interpretation of the results is improved and the computational time is hugely reduced.

As already commented, in the diffusion process, the studied phenomena are those related to slow diffusion, associated to cellularization, and fast diffusion, associated to vascularization. Assuming that the signal spectrum in a pixel $j$ can be expressed as a weighted sum of different decreasing exponential functions modeling the different phenomena of the diffusion process, we propose the following model:

$$
s_{j}=\sum_{i=1}^{I} c_{i j}\left(\alpha_{i} e^{-\beta_{i} b}\right) ; \quad \alpha_{i}, \beta_{i}, c_{i j} \geq 0
$$

Where $I$ stands for the number of exponential functions used. In this work, models using 1, 2 and 3 exponential functions are proposed. The triexponential approach is proposed in order to model a possible additional behavior with physiological meaning that might remain in the residuals of the biexponential approach.

The main differences regarding the theoretical models outlined in Section 1 are the nonormalization of the spectra (using $s$ instead of $\mathrm{s}_{\mathrm{s}} \mathrm{s}$ ) and the independence between the $\alpha_{i}$ coefficients, which are not forced to sum 1 . 
To analyze these data, Multivariate Curve Resolution in it Alternating Least Squares (MCR-ALS) version [9, 12] has been used due to its capability to obtain non-orthogonal behaviors and modelling additive phenomena. MCR-ALS is an iterative method that performs a bilinear decomposition of the $\mathbf{S}$ matrix by means of an alternating least squares optimization.

$$
\mathbf{S}=\mathbf{C D}^{\prime}+\mathbf{E}
$$

In diffusion studies matrix $\mathbf{S}$ contains the signal spectrum $\mathbf{s}$ for each pixel in rows, $\mathbf{D}^{\mathbf{P}}$ is a matrix containing in its rows each of the diffusion behaviors modeled, $\mathbf{C}$ gathers in its rows the relative contribution of each modeled behavior for each pixel of the image, and $\mathbf{E}$ is a residual matrix.

MCR-ALS focuses on describing the evolution of the experimental multicomponent measurements through their pure component contributions [13], without imposing hardto-accomplish constraints from a chemical, physical or biological interpretation point of view (e.g. orthogonality in the modeled behaviors)

However, the iterative process can provide infinite solutions for the same data matrix. This problem can be solved by imposing other type of constraints commonly related to prior knowledge about the problem faced, so that it is possible to obtain easier-tointerpret solutions, which also tend to be unique when the constraints introduced under the hypothesized assumptions are sensible [14]. In the case of the diffusion process, the following additional constraints can be applied successively in order to validate the theoretical models most commonly applied in clinical practice introduced in Section 1.

1. Non-negativity constraints are applied both in $\mathbf{D}$ and $\mathbf{C}$ matrices, because the modeled behaviors and their relative contribution in a pixel have to be nonnegative.

2. Unimodality constraints where only one maximum or minimum is admitted are imposed only with $\mathbf{D}$, due to the fact that the modeled behaviors are monotonically decreasing.

3. Shape constraints are applied in the $\mathbf{D}$ matrices in order to obtain a specific mathematical expression (i.e. exponential decay) for the modeled behaviors. 
The exponential decay shape constraints in the $\mathbf{D}$ matrix are introduced in the following way in the MCR-ALS algorithm [15]: for each one of the behaviors obtained from Model 2, initial parameters $\square$ and $\square$ are fitted using the exponential decays shown in eq. 3. Afterwards, using these exponential functions and the abscissa's b values, the "pure" $\mathbf{d}_{\text {initial }}$ values are predicted, and arranged in a $\mathbf{D}_{\text {inital }}$ matrix. This $\mathbf{D}_{\text {initial }}$ matrix is introduced in eq. 4 , and by running a one loop of the ALS algorithm, a new $\mathbf{D}$ matrix is obtained. Following the same procedure as the one used from $\mathbf{D}$ matrix of Model 2 a new $\mathbf{D}$ matrix is obtained, repeating the ALS sequence until convergence

Including these constraints, the model can select the solutions with more physiological meaning, discarding the rest lacking any clinical interpretation. This is a clear advantage over PCA when interpretability is of major importance, since the orthogonality in the latent variables imposed in PCA makes clinical interpretation of the modeled behaviors harder.

The sequential procedure to obtain the different MCR models follows:

1. Fit MCR models with only non-negativity constraints in $\mathbf{C}$ and $\mathbf{D}$ with one, two and three components.

Select the model with most appropriate number of components according to the similarity of the corresponding fitted behaviors with the exponential decay (i.e. expected physiological behavior). This is Model 0.

2. Fit a new MCR model including non-negativity constraints in $\mathbf{C}$ and $\mathbf{D}$, and unimodality constraints in $\mathbf{D}$ using the number of components decided in step 1. This is Model 1.

3. Check the adequacy of Model 1 by fitting the behaviors obtained (rows of $\mathbf{D}^{\prime}$ matrix in step 2) with an exponential function and projecting the predicted exponential behavior onto the original data matrix $\mathbf{S}$ to obtain the new $\mathbf{C}$ matrix. This is Model 2.

4. Finally, fit a new MCR model including non-negativity constraints in $\mathbf{C}$ and $\mathbf{D}$, and shape (i.e. exponential decay) constraints in $\mathbf{D}$ using the number of components decided in step 1. This is Model 3. 
The MCR models built this way provide the matrices D' and C previously commented, gathering at each pixel location the behaviors found and their relative contribution, respectively. By relocating the pixels in each column of $\mathbf{C}$ matrix into their original spatial dimension (192x192 pixels), new images known as score distribution maps are obtained, which permit to locate those pixels more related to each of the corresponding behaviors provided by the model. It must be stressed that these score distribution maps from $\mathbf{C}$ matrix, not the prostate diffusion behaviors modeled in D' matrix, are the ones performing as imaging biomarkers. This is a conceptual difference with the clinical theoretical models method, where the biomarkers (e.g. $D, D^{*}$ and $f$ in the case of a biexponential model) are extracted from the behavior modeled at each pixel location.

This tool can help the doctors to locate areas of the prostate that may be potentially affected by a tumoral process. Furthermore, the distribution maps of the Residuals Sum of Squares (RSS) were used as a validation method, as they show the pixels that are not well fitted by the model.

All calculations for the unfolding process and MCR modeling were done in Matlab (The Mathworks Inc., Natick, MA, USA) [12, 15].

\section{RESULTS}

\subsection{Model 0}

Initially, the only constraint considered is non-negativity. Figures 1 and 2 posted in supplementary material show the results with one and two components respectively. In the one-component model the fitted behavior follows an exponential decay pattern. As can be seen in the scores distribution map shown in the supplementary material (Fig. supp. 1a), the pixels related to this behavior are distributed in the whole image, being the residual sum of squares (RSS) low (Fig. supp. 1b). However, with two components, the residuals are even lower (the model explains $99 \%$ of the variability) and the behaviors are more physiologically meaningful because of the form of the obtained curves: a quick fall related to perfusion and a slow fall associated to real diffusion (Fig. supp. 2d). Nevertheless, there are still two "artifacts" (slight increases of signal 
intensity) present in both behaviors, at lower b-values in the pure diffusion (slow diffusion, d1) component and at higher b-values in the perfusion component (fast diffusion, d2). Regarding the distribution maps, they show (in the supplementary material) that the component related to diffusion (Fig. supp. 2a) is more representative in the whole zone of the prostate than that of the perfusion component (Fig. supp. 2b), which is more localized.

By comparing both models (with one and two components), it can be seen that the behavior fitted in the one-component model (Fig. supp. 1c) is a combination of the behaviors modeled in the two-component model (Fig. supp. 2d). This can also be observed in the score distribution maps (Fig. supp. 1a and Fig. supp. 2a-b). Therefore, the one-component MCR model is not able to explain all the physiological behaviors present in the diffusion studies due to its simplicity.

Supplementary material Fig. supp. 3 shows the modeled behaviors from the MCR model with three components. The behaviors associated to components 2 and 3 present shapes that make no sense in diffusion studies because the signal spectra cannot increase as the b-value goes up (it is assumed that signal always attenuates when the bvalue increases). Thus, in the following the two-components model is selected as the most appropriate to study the diffusion process.

\subsection{Model 1}

The next step consists of including new constraints in the model. The first of these are the unimodality ones (in D matrix) in order to provide new behaviors more similar to the exponentially decay shape. Actually, monotonicity constraints should be introduced, but this is accomplished in the following steps (with the shape constraints), so it was preferred to slowly adding constraints, in order to carefully validate the models. The inclusion of this type of constraint allows removing the artifact present in the perfusion component at high b-values (a slightly increase of $\mathrm{d} 2$ from 1500 to 2000 in the b-value) that appears in Model 0 (Fig. supp. 2d). Nevertheless, there is still one artifact (maybe introduced by the image acquisition system) at low b-values for the first behavior (diffusion component), which still accomplishes the unimodality constraint. After careful discussion with doctors, it was decided that this artifact could not happen from a 
physiological point of view. Furthermore, adding a new component did not isolate this behavior (results not shown).

\subsection{Model 2}

With the aim of removing the second artifact and checking the adequacy of the biexponential model in diffusion MR studies, a fitting of the behaviors is proposed using: (1) the results provided by Model 1 (the pure behaviors saved in the D matrix) and (2) the information of the clinical theoretical models that proposes two decreasing exponential behaviors. Therefore, using fitting algorithms, both behaviors are tuned to exponential expressions $\alpha \exp (-\beta b)$, that are the most accepted among the current research in DW-MRI, either by the model used to extract the ADC (eq. 1) or the model used to calculate D, D* and f from the IVIM theory (eq. 2). Both models have been applied in the study of diffusion behavior in several tissues and organs in the related diseases of the body [16-20]. Furthermore, the goodness of fit is high (95\%) obtaining two different pure exponential decays related to diffusion (d1) and perfusion (d2), respectively.

Once the exponential shape of the behaviors is obtained, the next step was to project the original data matrix on the pseudoinverse of the new fitted $\mathbf{D}$ to get the scores $\mathbf{C}$.

\subsection{Model 3}

Finally, once the biexponential model is validated, the shape constraints for the D matrix were included in the iterative process of the MCR-ALS algorithm, assuming classical exponential expressions $\alpha_{i} \exp \left(-\beta_{i} b\right)$ for both behaviors, and non-negativity for $\mathbf{D}$ and $\mathbf{C}$ matrices. For every case, the exponential parameters, $\alpha_{i}$ and $\beta_{i}(\mathrm{i}=1,2)$, obtained from Model 2 have been used as an initial approximation.

\section{[INSERT FIG. 3 ABOUT HERE]}

As it can be seen in Fig. 3d, the fitted behaviors are two different strictly decreasing exponentials, one related to diffusion (slow attenuation) and other related to perfusion 
(fast attenuation). Furthermore, the goodness of fit is at least $99 \%$ in all the cases under study, which derives in low RSS distribution maps (Fig. 3c).

\section{DISCUSSION}

The monoexponential model seems too simple when trying to obtain all the behaviors present in the images. The triexponential approach is not useful either, as it provides behaviors that are not physiologically interpretable. On the other hand, the biexponential model has shown up as a sensible approach for modeling the diffusion process. This model has been sequentially improved by comparing the new results provided by each further constrained model with the previous one. These conclusions are significantly related to the existing knowledge about the behavior of diffusion in tissues, where the bi-exponential approach through the IVIM theory is preferred in medical imaging research to explain the behavior of water molecules in tissues like tumors, where not only a cellular component, but also a micro-vascular behavior is appreciated.

Figure 4 summarizes the two modeled behaviors obtained from Models 1, 2 and 3 and Fig. supp. 5 shows the distribution maps for these models. In all three models, the fast diffusion component presents an exponential decay, even in Model 1 where no shape is imposed. Regarding the slow diffusion component, it also presents an exponential decay afterwards removing the artifact present at lows b-values (Models 2 and 3). This can be seen in the Fig. supp. 4 posted in supplementary material where the higher residuals of model 3 related to low b-values coincide with this artifact in model 1 . Therefore, the pixels mainly related to this phenomenon are those having higher (negative) residuals in model 3, this can be seen in Fig. supp. 5, third column, third row. On the other hand, the distribution maps observed in Fig. supp. 5 remain almost the same in all three models.

\section{[INSERT FIG. 4 ABOUT HERE]}

This way, the proposed procedure provides sensible results that complement the IVIM biexponential model, using the scores distribution maps as a quantification of each behavior gathered in D matrix at each pixel location. Still, both the MCR-based modeling and the theoretical biexponential modeling show two basic differences: first, 
the normalization of the spectra; and second, the fact that the two factors weighting the exponentials do not have to be necessarily complementary. Furthermore, the fitting of the IVIM model is fitted pixel-by-pixel, unable to take profit of the global information that can be obtained using chemometric models.

\section{CONCLUSIONS}

Along the present work, the appropriateness of the exponential theoretical models with a data driven model incorporating prior knowledge of the diffusion process has been accomplished. The monoexponential model has been discarded, whereas the biexponential IVIM model has been discussed.

This paper shows the capability Multivariate Curve Resolution (MCR) models to extract behaviors with physiological meaning from the DW-MRI images by including a priori knowledge. Furthermore, MCR lets to directly locate and grade the intensity of these behaviors in the images, providing new imaging biomarkers, complementary to those provided by the IVIM model, to improve clinical diagnosis.

\section{Acknowledgements}

The authors want to thank prof. Anna de Juan for her comments and help in using the software for this study. This research work was partially supported by the Spanish Ministry of Economy and Competitiveness under the project DPI 2011-28112-C04-02.

\section{REFERENCES}

[1] Charles-Edwards EM, De Souza NM. Diffusion-weighted magnetic resonance imaging and its application to cancer. Cancer Imaging. 2006; 6:135-143.

[2] Le Bihan D. Molecular diffusion nuclear magnetic resonance imaging. Magn. Reson. Q. 1991; 7:1-30.

[3] Lemke A, Stieltjes B, Schard LR, Faun FB. Toward an optimal distribution of b values for intravoxel incoherent motion imaging. Magn. Reson. Imaging. 2011; 29:766776. 
[4] Le Bihan D, et al. MR imaging of intravoxel incoherent motions: application to diffusion and perfusion in neurologic disorders. Radiology. 1986; 161:401-407.

[5] Geladi P, Grahn H. Multivariate Image Analysis. Wiley: Chichester, England, 1996.

[6] Prats-Montalbán JM, Ferrer A, de Juan A. Multivariate image analysis: a review with applications. Chemom. Intell. Lab. Syst. 2011; 107:1-23.

[7] Jackson JE. A User's guide to Principal Components. Ed. Wiley: New York, 1991.

[8] Prats-Montalbán JM, Sanz-Requena R, Martí-Bonmatí L, Ferrer A. Prostate functional magnetic resonance image analysis using multivariate curve resolution methods. J. Chemometrics. 2013; DOI: 10.1002/cem.2585

[9] Tauler R, Smilde AK, Kowalski BR. Selectivity, local rank, three-way data analysis and ambiguity in multivariate curve resolution. J. Chemometrics. 1995; 9: 31-58.

[10] Tauler R. Multivariate curve resolution applied to second order data. Chemom. Intell. Lab. Syst. 1995; 30:133-146.

[11] de Juan A, Tauler R. Chemometrics applied to unravel multicomponent processes and mixtures: revisiting latest trends in multivariate resolution. Anal. Chim. Acta 2003; 500: $195-210$.

[12] Jaumot J, Gargallo R, de Juan A, Tauler R. A graphical user-friendly interface for MCR-ALS: a new tool for multivariate curve resolution in MATLAB. Chemom. Intell. Lab. Syst. 2005; 76: 101-110.

[13] A. de Juan, R. Tauler, Multivariate curve resolution (MCR) from 2000: Progress in concepts and applications, Critical Reviews in Analytical Chemistry 36 (3-4) (2006) 163-176.

[14] Jaumot J, Tauler R. MCR-BANDS: A user friendly MATLAB program for the evaluation of rotation ambiguities in Multivariate Curve Resolution. Chemom. Intell. Lab. Syst. 2010; 103: 96-107.

[15] Multivariate curve resolution homepage, http://www.mcrals.info/.

[16] Riches SF, Hawtin K, Charles-Edwards EM, de Souza NM. Diffusion-weighted imaging of the prostate and rectal wall: comparison of biexponential and monoexponential modelled diffusion and associated perfusion coefficients. NMR Biomed. 2009; 22(3): 318-325.

[17] Rheinheimer S, Stieltjes B, Schneider F et al. Investigation of renal lesions by diffusion-weighted magnetic resonance imaging applying intravoxel incoherent motion-derived parameters--initial experience. Eur J. Radiol 2012; 81(3): 310-316. 
[18] Lemke A, Laun FB, Klauss M et al. Differentiation of pancreas carcinoma from healthy pancreatic tissue using multiple b-values: comparison of apparent diffusion coefficient and intravoxel incoherent motion derived parameters. Invest Radiol. 2009; 44(12): 769-775.

[19] Federau C, Maeder P, O'Brien K, Browaeys P, Meuli R, Hagmann P. Quantitative measurement of brain perfusion with intravoxel incoherent motion MR imaging. Radiol. 2012; 265(3):874-881.

[20] Sigmund EE, Cho GY, Kim S et al. Intravoxel incoherent motion imaging of tumor microenvironment in locally advanced breast cancer. Magn Reson Med. 2011; 65(5):1437-1447. 


\section{Figure Captions}

Figure 1. Signal attenuation spectra in a DW-MRI case. (a) Before normalization and (b) after normalization.

Figure 2. 3D matrix for a particular slice of a patient (a); 2D unfolded matrix (b).

Figure 3. MCR model 3 (99\% of explained variability). (a) Score distribution map related to d1 (slow diffusion, solid blue line). (b) Score distribution map related to $\mathrm{d} 2$ (fast diffusion, dashed green line). (c) RSS distribution map. (d) Behaviors provided by the MCR model.

Figure 4. Comparison between the behaviors provided by models 1 (solid red line), 2 (dashed blue line) and 3 (dotted black line). 